\title{
Ayushman Bharat: Dental Perspective
}

\section{Annapoorna HB and Dipayan D*}

Department of Public Health Dentistry, Triveni Institute of Dental Sciences, India

*Corresponding author: Dipayan Datta, Senior Lecturer, Department of Public

Health Dentistry, Triveni Institute of Dental Sciences, Hospital and Research Centre, Chhattisgarh, India, Tel: 7358565184, Email: datta.dipayan2@gmail.com

\section{Editorial}

Volume 3 Issue 4

Received Date: October 22, 2018

Published Date: October 30, 2018

DOI: 10.23880 /oajds- 16000203

\section{Editorial}

"Ayushman bhava" is the frequently heard blessing from the elders in India which means 'live long' and this can be attained as well as enjoyed only with a healthy life. The term 'Ayushman Bharat' simply reflects the purpose of the scheme that is to make India a healthier nation by empowering its health care system to reach the highest level like the way India's economy has grown to one of the largest ones in the world.

Among the 1.3 billion people of this developing nation, nearly $70 \%$ are from rural areas and 30\% from urban. People are facing difficulties to achieve good health because of inadequate access, limited coverage of health services and increased out-of-pocket expenditure for treatment. The situation is even worse for the people from the extremely rural areas who cannot afford the high cost of medical treatment. To overcome the health disparity in different parts of this country and to cover most of the population in disseminating proper health services, the Ayushman Bharat or Pradhan Mantri Jan Arogya Yojana, 2018 has been launched which comprises two schemes - firstly to develop 150,000Health and Wellness Centres across the country to provide universal health-care coverage and secondly to cover over 10 crore poor families based on socio-economic caste census database to provide up to yearly 5 lakh rupees to each family for secondary and tertiary health care. This scheme will be in action through Health and Wellness Centres within the primary health centre or sub-centre in the village and that will be providing preventive, promotive, and curative care for non-communicable diseases, psychiatric care, geriatric care, palliative care along with dental care. These centres are expected to be equipped with basic diagnostic devices and be connected to the district hospital for advanced medical care.
From the perspective of dental health care, after National Health Policy (2017) put light on the significance of oral health, Ayushman Bharat continues it to help the improvement of the oral health care of the entire nation. Dental problems are becoming one of the major public health concerns in India. Dental problems are not only related to pain, masticatory problems, and aesthetic problems but also lead to the loss of working man-hours affecting the quality of life. On one side, lack of awareness about oral hygiene is leading to increased prevalence of gingival and periodontal diseases among people from all age groups and on the other side, excessive sugar consumption is resulting in high dental caries prevalence among the younger generation. In spite of the ban on tobacco products, consumption of tobacco in both smoking and smokeless forms has increased in the recent years leading to oral precancers and cancers emerging as a major threat to an alarming proportion among the Indian population.

Under such circumstances, Ayushman Bharat is undoubtedly a noteworthy step taken by the Government of India. On one side, the Health and Wellness Centres will be there to take care of the patients with various dental problems and on the other side, the insurance will support the poor patients to receive advanced dental treatments which they could never afford. One added advantage of the scheme is that the beneficiaries can access the required services anywhere in India, for example, an eligible family from Chhattisgarh can receive the necessary treatment even in West Bengal. Ayushman Bharat scheme has the capability in empowering the health system by enhancing its efficiency, betterment of the health outcome and rising patient satisfaction. Once it is in operation under proper regulation, monitoring and continued evaluation the dream of a healthier India will definitely come true. 